\title{
Recent advances in somatic gene therapy for hereditary respiratory diseases
}

\author{
C A Owen
}

Cystic fibrosis and $\alpha_{1}$ antitrypsin deficiency are the two most common potentially lethal hereditary diseases of white people. Disease of the respiratory tract is the most common cause of morbidity and mortality in both. The genetic defects underlying these disorders are now well understood, and there is enthusiasm for the possibility of somatic gene therapy. This review will summarise the recent experiments exploring the feasibility of somatic gene therapy for cystic fibrosis and $\alpha_{1}$ antitrypsin deficiency and will also outline the substantial obstacles yet to be overcome.

\section{What is somatic gene therapy?}

Somatic gene therapy requires the introduction of a normal recombinant gene into the somatic cells of an individual with a hereditary disorder to reconstitute specific gene products and their functions. The most common approach to gene therapy is to transfer genes to target cells by means of retroviruses, which have been modified in such a way that they are devoid of genes coding for viral proteins or malignant transformation. ${ }^{1}$ The gene of interest is inserted into the viral genome and target cells isolated from the host are infected with the recombinant vector in vitro, which results in the stable integration of the gene of interest into the host cell genome. The infected host cells are then transplanted into the host.

\section{Applications of somatic gene therapy CYSTIC FIBROSIS}

The normal cystic fibrosis gene can be transferred to mammalian cell lines in vitro. Drumm et $a l^{2}$ used a retroviral vector to transfer the normal gene into a pancreatic adenocarcinoma cell line derived from a patient with cystic fibrosis, which expressed the chloride transport abnormality characteristic of this disease. The infected pancreatic cells expressed the normal cystic fibrosis protein (cystic fibrosis transmembrane conductance regulator), which was associated with the correction of the chloride secretory abnormality. Similar results have been obtained with cultured airway epithelial cells from patients with cystic fibrosis. ${ }^{3}$ The cystic fibrosis gene has not, however, yet been transferred to live animals and the efficacy and safety aspects of somatic gene therapy for patients with cystic fibrosis have yet to be addressed.

\section{$\alpha_{1}$ ANTITRYPSIN DEFICIENCY}

Retroviral vectors have also been used to transfer human $\alpha_{1}$ antitrypsin cDNA to cultured murine fibroblasts. The transformed fibroblasts secreted human $\alpha_{1}$ antitrypsin that showed antiprotease activity against neutrophil elastase, the major physiological target of $\alpha_{1}$ antitrypsin. ${ }^{45}$ One group also transplanted the transformed fibroblasts into the peritoneal cavities of nude mice, and human $\alpha_{1}$ antitrypsin was detected subsequently both in serum and in the epithelial surface of the lungs. ${ }^{5}$

An alternative method of transferring genes to treat hereditary respiratory diseases is to deliver the recombinant vector directly to the respiratory tract by tracheal instillation. ${ }^{6}$ One potential problem in using retroviral vectors to transfer genes to humans by the tracheal route is that these vectors require proliferation of the target cells for expression of the transferred protein, ${ }^{7}$ whereas a large proportion of respiratory epithelial cells are terminally differentiated and are therefore incapable of proliferation. ${ }^{8}$ There are also potential dangers in administering retroviral vectors to humans. For example, retroviruses are integrated randomly with the host chromosome and may become integrated with a normal gene of the host, creating a new mutation by insertional mutagenesis. ${ }^{1}$ In addition, recombinant retroviruses may recombine spontaneously with retroviruses in the environment, giving rise to new infectious viruses of unknown pathological potential. ${ }^{1}$

Perhaps the best hope for successful gene therapy for hereditary respiratory diseases lies in the use of adenoviral vectors. Adenovirus mediated transfer of functional genes circumvents the problem of host cell proliferation as adenoviruses do not require host cell proliferation for expression of adenoviral proteins and these viruses are also trophic for respiratory epithelium. ${ }^{9}$ Furthermore, although human infection with adenovirus is common, it has not been associated with the subsequent development of serious sequelae.

Recently Rosenfeld et al used an adenovirus vector to transfer the human $\alpha_{1}$ antitrypsin gene to cotton rats by the tracheal route. ${ }^{6}$
Pulmonary Medicine, University of Utah UT84132, USA C A Owen

Reprint requests to: Dr Owen 
These investigators constructed the recombinant adenovirus vector containing the human $\alpha_{1}$ antitrypsin gene by deleting the sequences of the wild type adenoviral type 5 genome that are essential for viral replication and inserting human $\alpha_{1}$ antitrypsin CDNA in their place. The virus therefore remained capable of infecting cells, but was incapable of replication, host cell lysis, and infection of other host cells.

In preliminary in vitro experiments epithelial cells obtained from cotton rat lungs were cultured and infected with the adenovirus vector. Human $\alpha_{1}$ antitrypsin messenger RNA was detected in the rat epithelial cells 24 hours after infection with the vector. In addition, human $\alpha_{1}$ antitrypsin protein was detected in the supernatants of the cells infected with the adenovirus vector, indicating that the human $\alpha_{1}$ antitrypsin transcripts were capable of directing the synthesis and secretion of human $\alpha_{1}$ antitrypsin.

In subsequent in vivo experiments the adenovirus vector was instilled directly into the trachea of cotton rats and lung tissue was harvested from the rats at intervals for up to one week. De novo synthesis and secretion of human $\alpha_{1}$ antitrypsin was found in lung tissue harvested from infected rats, and synthesis of the protein lasted up to one week. The secreted human $\alpha_{1}$ antitrypsin protein was functional because it formed a complex with human neutrophil elastase. Expression of the human $\alpha_{1}$ antitrypsin gene was limited to respiratory epithelial cells, however, and the distribution of transformed cells was patchy. Furthermore, only trace amounts of human $\alpha_{1}$ antitrypsin were detected in the epithelial lining fluid of rats infected with the adenovirus vector.

\section{Obstacles}

There are several obstacles to be overcome before somatic gene therapy can be applied to the management of human hereditary respiratory diseases, including the inability to regulate transferred genes and uncertainties about the safety of administering recombinant viruses to humans. Moreover, if we are to show a benefit from somatic gene therapy efficacy studies will be prolonged and expen- sive, and will require large numbers of patients.

With respect to gene therapy for $\alpha_{1}$ antitrypsin deficiency, the safety aspects are critical because the natural history of this condition is largely unknown, and many patients appear to escape seriously disabling disease, particularly if they do not smoke. ${ }^{10}$ In addition, sufficient quantities of $\alpha_{1}$ antitrypsin must be produced by lung cells in order to slow the rate of progression of lung disease. Intratracheal administration of recombinant adenoviral vectors may have greater potential in the management of cystic fibrosis, which is a disease of the airways.

In summary, pioneering steps have been taken towards somatic gene therapy for two of the most prevalent serious hereditary pulmonary conditions of white people. Although the rate of progress in rapidly developing fields such as recombinant DNA technology is difficult to predict, clinical applications will probably be in the distant future.

1 Ledley FD. Somatic gene therapy for human disease: Background and prospects. Part 1. J Pediatr 1987;110: $1-8$.

2 Drumm ML, Pope HA, Cliff WH, Rommens JM, Marvin SA, Tsui LC, et al. Correction of the cystic fibrosis defect in vitro by retrovirus-mediated gene transfer. Cell 1990; 62:1227-33.

3 Rich DP, Anderson MP, Gregory RJ, Cheng SH, Paul S, Jefferson DM, et al. Expression of cystic fibrosis transmembrane conductance regulator corrects defective chloride channel regulation in cystic fibrosis airway epithelial cells. Nature 1990;347:358-63.

4 Ledley FD, Grenett HE, Bartos DP, Woo SLC. Retroviral mediated transfer and expression of human alpha antitrypsin in cultured cells. Gene 1987;61:113-8.

5 Garver RI, Chytil A, Courtney M, Crystal RG. Clonal gene therapy: transplanted mouse fibroblast clones express human $\alpha_{1}$-antitrypsin gene in vivo. Science 1987;237: $762-4$.

6 Rosenfeld MA, Siegfried W, Yoshimura K, Yoneyama K, Fukayama $M$, Stier LE, et al. Adenovirus-mediated transfer of a recombinant $\alpha_{1}$-antitrypsin gene to the lung epithelium in vivo. Science 1991;252:431-4.

7 Miller DG, Adam MA, Miller AD. Gene transfer by retrovirus vectors occurs only in cells that are actively replicating at the time of infection. Mol Cell Biol 1990; replicating

8 Evans MJ, Shami SG. In: Lenfant C, Massaro D, eds. Lung cell biology. New York: Dekker, 1989:1-36.

9 Berkner KL. Development of adenovirus vectors for the expression of heterologous genes. Biotechniques 1988;6: 616-29.

10 Silverman EK, Pierce JA, Province MA, Rao DC, Campbel EJ. Variability of pulmonary function in $\alpha_{1}$-antitrypsin deficiency: clinical correlates. Ann Intern Med 1989:;111: 982-91. 